\title{
The assessment of somatosensory cortex plasticity during sleep deprivation by paired associative stimulation
}

\author{
M. GORGONI', F. FERLAZZO', A. D'ATRI', G. LAURI', M. FERRARA², \\ P.M. ROSSINI ${ }^{3,4}$, L. DE GENNARO'
}

'Department of Psychology, "Sapienza" University of Rome, Rome, Italy; ${ }^{2}$ Department of Life,
Health and Environmental Sciences, University of L'Aquila, L'Aquila, Italy; ${ }^{3}$ Institute of Neurology,
Catholic University of The Sacred Heart, Rome, Italy; " IRCCS San Raffaele Pisana, Rome, Italy

A B S T R A C T

Many animal studies suggest that during sleep deprivation (SD) synaptic strength should progressively increase, leading to the saturation of the ability to induce long-term potentiation (LTP). Nevertheless, direct evidences about the effects of sustained wakefulness on cortical plasticity in humans are still lacking. The aim of the present study was to assess changes in the ability to induce LTP-like mechanism in humans during a period of SD by means of a paired associative stimulation (PAS) protocol, which combines median nerve stimulation with transcranial magnetic stimulation (TMS) applied over the contralateral somatosensory cortex. During a 41-h SD protocol, 16 healthy subjects, defined as responders to the PAS protocol after a pre-selection session, were involved in 4 experimental sessions (11.00 a.m. and 11.00 p.m. of first and second day) with: a) pre-PAS somatosensory evoked potentials (SEPS) recordings; b) PAS protocol; c) post-PAS SEPs recordings. The effect of PAS on SEPs early components (N20-P25 complex) was assessed. During the first experimental session (without SD) no significant PAS effects on SEPs components amplitude have been found, and large intra- and inter-individual variability have been observed. A lack of significant changes has been observed also in the subsequent sessions. Our results index a low intra- and inter-individual reliability of the PAS protocol, suggesting particular caution when longitudinally evaluating the effect of this technique on cortical plasticity.

\section{Key words}

Sleep deprivation $\bullet$ Paired associative stimulation $\bullet$ Synaptic homeostasis $\bullet$ Cortical plasticity $\bullet$ Somatosensory evoked potentials $\bullet$ Transcranial magnetic stimulation

\section{Introduction}

The hypothesis that sleep and neural plasticity are strongly related is widely accepted. It is wellknown that post-learning sleep enhances memory consolidation (Gais et al., 2000; Maquet, 2001; Gais and Born, 2004; Walker and Stickgold, 2004; Stickgold, 2005). Moreover, the induction of plastic changes during wakefulness produces coherent and topographically specific changes during subsequent sleep in the principal marker of sleep intensity, that is the slow-wave activity (SWA). In fact, training with a visuomotor task (Huber et al., 2004), and the induction of a localized potentiation of the responses evoked by transcranial magnetic stimulation (TMS) (Huber et al., 2007) during wake is causally associated to a local increase of sleep SWA, and visual perceptual learning produces a local initiation of slow waves during NREM sleep that predicts subsequent skill improvement (Mascetti et al., 2013). 
On the contrary, diurnal arm immobilization leads to a local SWA decrease during sleep (Huber et al., 2006). Such changes should be modulated by plastic modification in terms of long-term potentiation (LTP) and long-term depression (LTD). In fact, also the direct production of LTP-like and LTD-like mechanisms during wake, by means of a TMS paired associative stimulation (PAS) protocol, induces localized transient modification in SWA during subsequent sleep (Bergmann et al., 2008; De Gennaro et al., 2008; Huber et al., 2008).

These findings are in line with the hypothesis that the principal role of sleep is the restoration of the synaptic homeostasis (Tononi and Cirelli, 2003; 2006; 2014). Wakefulness would be related with a progressive increase of the synaptic strength, with a cost in terms of energy consumption and cellular stress, provoking a reduction of neuronal selectivity and a saturation of learning ability. On the other side, sleep (and in particular SWA) would induce a synaptic renormalization process, that is a downscaling of synaptic strength, with a consequent restoration of neuronal selectivity and learning ability.

According to this assumption, sleep deprivation (SD) should be associated with a progressive increase of synaptic strength, leading to the saturation of the ability to induce LTP. Actually, a learning impairment is a typical consequence of prolonged wakefulness (Durmer and Dinges, 2005; Walker, 2008; McCoy and Strecker, 2011), and many in vitro studies show an inhibition of the hippocampal LTP and an enhancement of the LTD after sleep loss (Campbell et al., 2002; McDermott et al., 2003; Kopp et al., 2006). Prolonged wakefulness induces an increase in number and size of the central synapses in Drosophila melanogaster, and their reduction is possible only after sleep (Bushey et al., 2011; Donlea et al., 2011). A post-wakefulness increase and a post-sleep decrease of cortical synaptic efficacy (in terms of amplitude and frequency of miniature excitatory postsynaptic currents) have been observed in cats and mice cortical slices (Liu et al., 2010). Moreover, Vyazovskiy and co-workers (2008) have found in sleep-deprived rats an increase of amplitude and slope of local field power (a measure of synaptic strength), cortical excitability (2013), and cortical neurons firing frequency (2009). In humans, different studies have found an increase of cortical excitability after SD, in terms of motor evoked potentials (De Gennaro et al., 2007; Kreuzer et al., 2011) and TMS-evoked potentials (Huber et al., 2013), interpreted as an index of increased synaptic strength (Huber et al., 2013), while others found no SD-induced modulation of cortical excitability (Manganotti et al., 2001; 2006) or provided conflicting results (Civardi et al., 2001). In most cases the assessment of post-SD cortical excitability has been limited to frontal and prefrontal areas. An increase in the somatosensory cortex excitability after prolonged wakefulness has been observed, but the authors did not consider the possibility of the influence of a circadian modulation (Terney et al., 2005). Finally, a direct evidence that the effects of prolonged wakefulness on human cortical excitability are mediated by changes in LTP/LTD processes is still lacking: the modification in the ability to induce plastic mechanisms in humans after SD has never been directly investigated.

In a recent study (Gorgoni et al., 2014a) we have reported on the effects of a 41-h SD protocol on the excitability of the human somatosensory cortex, showing a progressive increase of cortical excitability with time awake, without any influence of the circadian factor and positively related to post-SD changes in subjective and behavioural sleepiness. With the aim to understand if SD-induced changes in somatosensory cortex excitability are associated with alteration of LTP mechanisms, we have also assessed in the same sample (during the same SD protocol) the effect of sustained wakefulness on cortical plasticity. In the present paper, then, we report on the effect of SD on the ability to induce LTP-like mechanisms in the somatosensory cortex. Cortical plastic processes have been produced by means of a PAS protocol, combining a single electric stimulus delivered at specific time intervals to a peripheral nerve with a single TMS pulse on the contralateral cerebral cortex. This protocol has been shown to induce LTP-like phenomena, which are persistent, topographically specific, and reversible (Stefan et al., 2000). It also requires the activation of NMDA receptors (Stefan et al., 2002; Wolters et al., 2003). PAS-dependent plastic changes have a cortical origin (Stefan et al., 2000; Wolters et al., 2005; Di Lazzaro et al., 2009a; 2009b), are dosedependent (Nitsche et al., 2007), and are suppressed by antagonists of the major neuromodulatory neurotransmitter systems (Korchounov and Ziemann, 
2011). The direction of cortical plasticity induced by paired stimulation depends on the interval between the stimuli. If sensory input from the median nerve $(\mathrm{MN})$ stimulation reaches the post-central cortex at appropriate intervals prior to magnetic stimulation (20-25 msec), PAS potentiates the local cortical plasticity. Conversely, if the magnetic stimulation precedes the arrival of the sensory input to the cortex, PAS can induce a LTD-like effect (Wolters et al., 2005; Murakami et al., 2008). Albeit Kriváneková and co-workers (2011) concluded that the effects of PAS on S1 are characterized by a large variability across individuals, different authors have induced coherent plastic changes by stimulating S1 (Wolters et al., 2005; Litvak et al., 2007; Pellicciari et al., 2009). In line with the synaptic homeostasis hypothesis (Tononi and Cirelli, 2003; 2006; 2014), an increase of LTP-like mechanisms during the SD protocol, which should progressively reach a saturation level, was expected.

\section{Methods Subjects}

Sixteen healthy male volunteers took part in the experiment (mean age $\pm \mathrm{SE}=23.3 \pm 0.64$ years). All subjects self-reported as right-handed and without history of central or peripheral neurological impairment. Exclusion criteria were: brain injury, alcohol abuse, diabetes, drug addiction, and contra-indications to TMS (Wassermann, 1998). Inclusion criteria were: normal sleep duration (habitual sleep time: 24.00$8.00 \pm 1 \mathrm{~h}$ ) and schedule, no daytime nap habits, no excessive daytime sleepiness, no other sleep, medical or psychiatric disorder, as assessed by a one-week (mean $\pm \mathrm{SE}=7 \pm 0.3$ days) sleep $\log$, the score on the Italian version of the Pittsburgh Sleep Quality Index [PSQI - (Curcio et al., 2013)], and a clinical interview. Since the PAS protocol is characterized by a large interindividual variability (Huber et al., 2008; Pellicciari et al., 2009; López-Alonso et al., 2014), a further inclusion criterion was the presence of a clear N20-P25 complex amplitude increase as a consequence of the PAS protocol, as assessed by a preliminary evaluation which preceded the current study. Pellicciari and co-workers (2009), after PAS intervention on a group of young subjects, found a mean N20-P25 amplitude increase of 5.4\%.
According to this result, in the present study a postPAS amplitude increase of the N20-P25 complex of at least $5.4 \%$ was used as inclusion criterion. We originally evaluated 39 subjects. The 16 selected subjects (41\% of the original group) showed an average N20-P25 amplitude increase of $27.4 \%$ during the pre-selection session.

Participants were required to avoid napping; actigraphic recordings (AMI Mini motion logger) were collected for about one week (mean $\pm \mathrm{SE}=7 \pm 0.3$ days) before the beginning of the experimental procedure to control subjects' compliance.

All subjects gave their written informed consent. The study was approved by the Institutional Ethics Committee of the Department of Psychology of the University of Rome "Sapienza", and was conducted in accordance with the Declaration of Helsinki.

\section{Procedure}

\section{Study design}

Figure 1 depicts the timeline diagram of the experimental protocol. On the morning of the experiment participants woke up on average at 7.00 a.m. (mean $\pm \mathrm{SE}=6.54 \pm 0.13$ a.m. based on sleep $\log$ ), and arrived at the laboratory at 9.00 a.m. for the electrodes montage. Experimental procedure started at 11.00 a.m. Subjects were evaluated in four different sessions carried out at the same time (11.00 a.m. and 11.00 p.m.) of the first and second day. Each session included: a) subjective sleepiness recordings; b) EEG recordings (5-min eyes-open condition); c) pre-PAS SEPs recordings; d) PAS protocol; e) post-PAS SEPs recordings; f) behavioural sleepiness recordings. We used this fixed sequence due to the relatively small sample size and to the main focus being on the electrophysiological measures. Methods and results concerning EEG, cortical excitability, subjective, and behavioural sleepiness measures have been reported elsewhere (Gorgoni et al., 2014a; 2014b).

During the experimental sessions, participants were seated on a comfortable chair in a soundproof, electrically shielded room. They were required to keep their right arm completely relaxed during the entire experimental session. When not involved in testing sessions, subjects were allowed to carry out their own preferred activities, such as reading, writing, listening to music, watching TV or playing games, always 


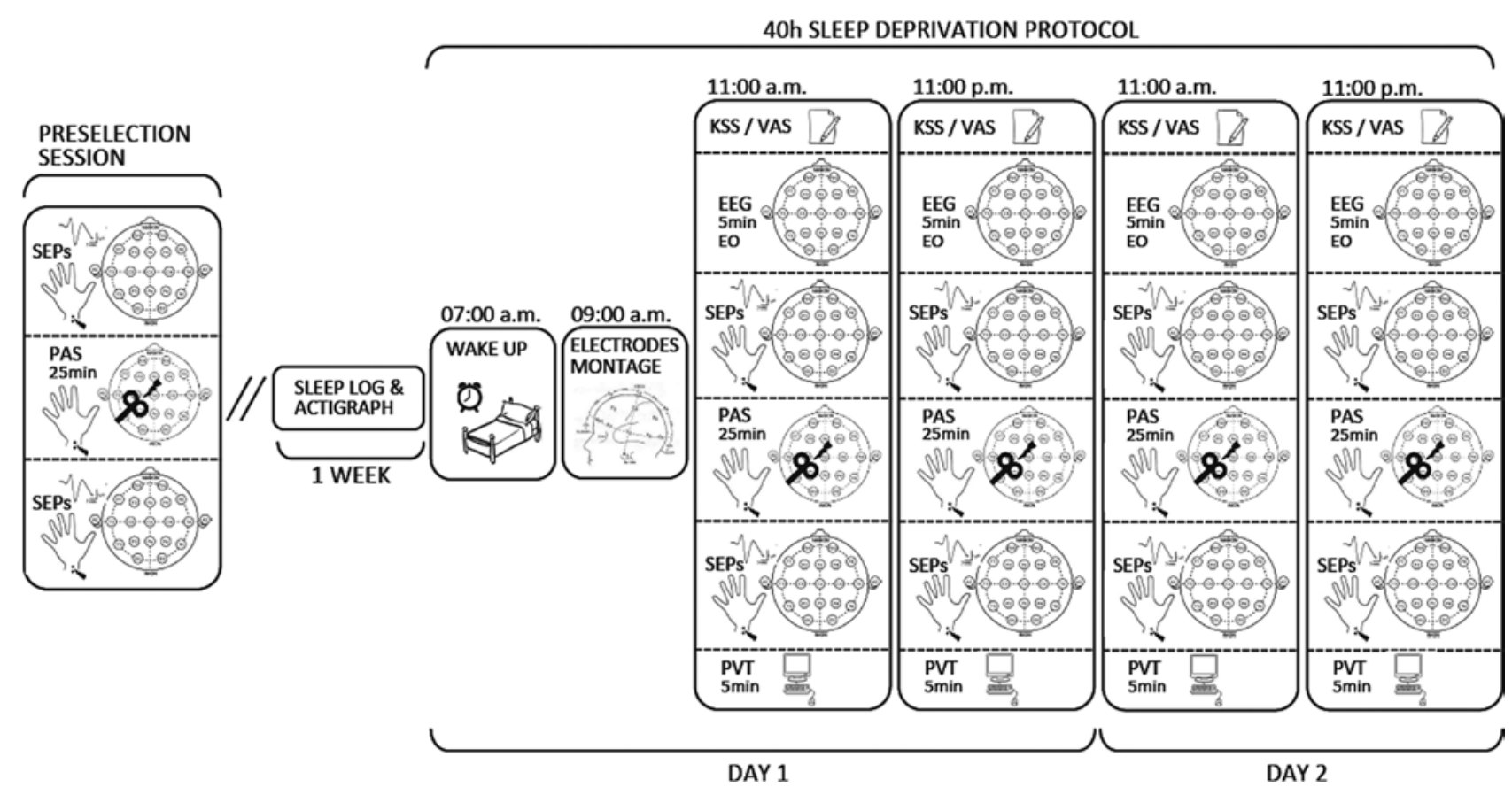

Fig. 1. - Timeline diagram of the experimental protocol. Subjects perform a pre-selection session about two weeks before the beginning of the experiment, with: 1) pre-PAS SEPs recordings; 2) PAS protocol; 3) post-PAS SEPs recordings. Only subjects showing an N20-P25 amplitude increase of at least $5.4 \%$ were recruited for the experiment. During the week preceding the beginning of the SD period, subjects were monitored by an actigraphic recording and sleep log. On the morning of the experiment subjects on average woke up at $7.00 \mathrm{a} . \mathrm{m}$. (mean $\pm \mathrm{SE}=6.54 \pm 0.13 \mathrm{based}$ on sleep $\mathrm{log}$ ) and went in the laboratory at $9.00 \mathrm{a} . \mathrm{m}$. for the electrodes montage. Experimental procedure started at 11.00 a.m. Subjects were evaluated in four different sessions carried out at the same time (11.00 a.m. and 11.00 p.m.) of the first and second day. Each session was conducted in the following sequence: a) subjective sleepiness recordings (Karolinska Sleepiness Scale and Visual Analog Scale for Global Vigor); b) EEG recordings (5-min eyes-open condition); c) pre-PAS SEPs recordings; d) PAS protocol; e) post-PAS SEPs recordings; f) behavioural sleepiness recordings (Psychomotor Vigilance Task). The 41-h schedule of sleep deprivation ended at midnight on the second day.

under the direct supervision of at least one experimenter. Lying down, sleeping, and vigorous physical activity were not permitted. Meals were provided to subjects at 08.30 a.m., 02.30 p.m. and 07.30 p.m. Non-scheduled light snacks were permitted, while caffeinated beverages, chocolate, alcohol, and medications that can influence sleepiness were not allowed during the deprivation protocol. Time information was available to subjects, and light exposure was not strictly controlled for (although the laboratory was constantly illuminated by 4 neon lamps, blinds only in part attenuated the light coming from the outside). The 41-h schedule of SD ended at midnight on the second day.

\section{Electrical somatosensory stimulation}

Electrical nerve stimulation was performed with a bipolar electrode (cathode proximal), connected to an electromyography (MYto, EBNeuro, Italy). The stimulating electrode was placed on the right $\mathrm{MN}$ at the level of the wrist (cathode proximal). MN stimulation was performed using a pulse width of $200 \mu$ s at a frequency of $3 \mathrm{~Hz}$ and a stimulation intensity of $\sim 300 \%$ of the individual perceptual threshold (Wolters et al., 2005). The hand representation at primary somatosensory cortex ("somatosensory hotspot") was marked $2 \mathrm{~cm}$ posterior to $\mathrm{C} 3$ position, corresponding to $\mathrm{Cp} 3$ (Wolters et al., 2005). Before and after PAS intervention, the responses to 500 electric stimuli were recorded and averaged (500 pre-PAS +500 post-PAS). The average perceptual threshold was $1.63 \mathrm{~mA}(\mathrm{SE}= \pm 0.05)$. The stimulating electrode was removed after every experimental session, and its position was marked with a soft-tip pen. Impedances were checked before the beginning of each session.

\section{SEPs Recordings}

An Esaote Biomedica VEGA 24 polygraph was used for SEPs recordings. EEG signals were recorded from 20 unipolar scalp derivations (see Gorgoni et 


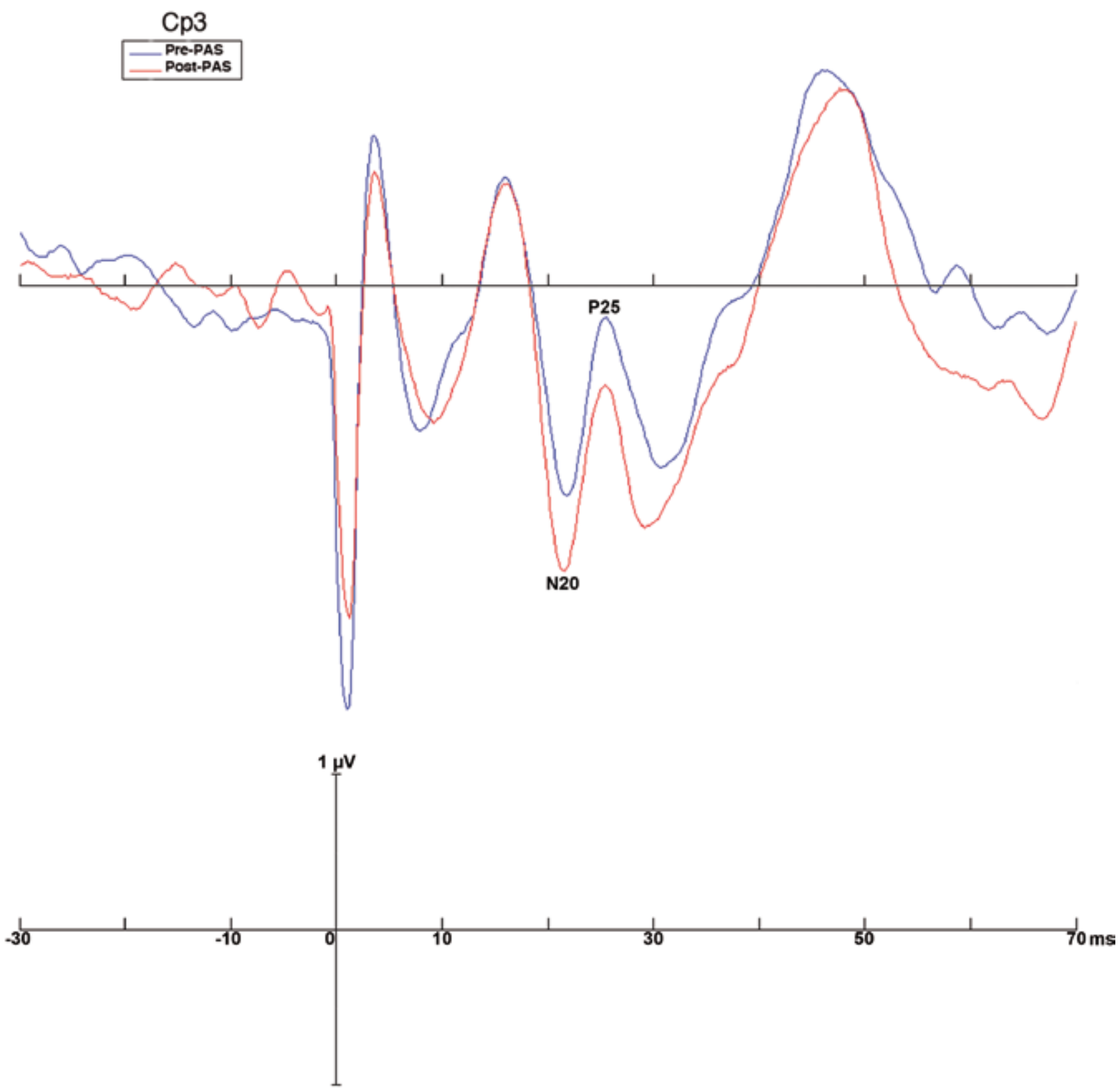

Fig. 2. - Pre- and post-PAS SEPs following right median nerve stimulation during the first experimental sessions at Cp3 in a representative subject.

al., 2014a for details) with averaged earlobes reference (A1, A2), using $\mathrm{Ag} / \mathrm{AgCl}$ sintered ring electrodes mounted on an elastic cap (EasyCap GmbH, Herrsching, Germany). For the purpose of this part of the experiment, only $\mathrm{Cp} 3, \mathrm{C} 3$ and $\mathrm{P} 3$ electrodes have been considered. Horizontal eye movements were detected by recording electrooculograms (EOGs) in order to reject, off-line, trials with ocular artefacts. Electromyogram (EMG) was recorded by two submental electrodes. During SEPs recordings EEG,
EOG and EMG signals were acquired at a sampling rate of $5 \mathrm{kHz}$ and band-pass filtered at $0.03-1500 \mathrm{~Hz}$. The skin-electrode impedance was kept below $5 \mathrm{k} \Omega$. EEG, EOG and EMG electrodes were not removed after any recording session, and impedances were checked before the beginning of each session.

Surface EMG activity was recorded from the right APB muscle with the active electrode mounted on the belly muscle and the reference electrode placed over the base of the metacarpophalangeal 


\begin{tabular}{|c|c|c|c|c|c|c|c|c|}
\hline \multirow[b]{2}{*}{ Deriv. } & \multicolumn{2}{|c|}{$\begin{array}{c}1^{\text {st }} \text { experimental session } \\
\left(11.00 \text { a.m. }-1^{\text {st }} \text { day }\right)\end{array}$} & \multicolumn{2}{|c|}{$\begin{array}{l}2^{\text {nd }} \text { experimental session } \\
\text { (11.00 p.m. }-1^{\text {st }} \text { day) }\end{array}$} & \multicolumn{2}{|c|}{$\begin{array}{l}3^{\text {rd }} \text { experimental session } \\
\text { (11.00 a.m. }-2^{\text {nd }} \text { day) }\end{array}$} & \multicolumn{2}{|c|}{$\begin{array}{l}4^{\text {th }} \text { experimental session } \\
\text { (11.00 p.m. }-2^{\text {nd }} \text { day) }\end{array}$} \\
\hline & Pre-PAS & Post-PAS & Pre-PAS & Post-PAS & Pre-PAS & Post-PAS & Pre-PAS & Post-PAS \\
\hline $\mathrm{Cp} 3$ & $2.86 \pm 0.36$ & $3.00 \pm 0.43$ & $3.16 \pm 0.41$ & $2.85 \pm 0.41$ & $3.27 \pm 0.49$ & $3.10 \pm 0.43$ & $3.36 \pm 0.51$ & $3.28 \pm 0.5$ \\
\hline $\mathrm{C} 3$ & $2.36 \pm 0.37$ & $2.44 \pm 0.38$ & $2.49 \pm 0.38$ & $2.25 \pm 0.42$ & $2.61 \pm 0.42$ & $2.44 \pm 0.42$ & $2.68 \pm 0.48$ & $2.56 \pm 0.51$ \\
\hline P3 & $2.69 \pm .037$ & $2.75 \pm 0.44$ & $2.92 \pm 0.42$ & $2.66 \pm 0.41$ & $3.15 \pm 0.51$ & $2.99 \pm 0.49$ & $3.12 \pm 0.57$ & $3.10 \pm 0.52$ \\
\hline
\end{tabular}

joint of the thumb. Active and reference electrodes were removed after every experimental session and their positions were marked with a soft-tip pen. Impedances were checked before the beginning of each session. During SEPs recordings subjects were asked to keep their eyes open and to fixate a point on the wall.

\section{Transcranial magnetic stimulation}

TMS was applied using a Magstim 200 mono-phasic magnetic stimulator connected to a Bistim module and a figure-of-eight coil with a $9 \mathrm{~cm}$ external diameter (Magstim Company Limited, UK). The peak magnetic field produced by such a coil is 2.0 $\mathrm{T}$. The coil was placed tangentially to the scalp with the handle pointing backwards and laterally at about a $45^{\circ}$ angle away from the midsagittal axis of the subject's head. The optimal site of stimulation for eliciting motor-evoked potentials (MEPs) in the right abductor pollicis brevis (APB), termed the "motor hotspot", was chosen by positioning the coil approximately over the central sulcus and moving it on the scalp in $0.5 \mathrm{~cm}$ steps over M1 of the left cortex, assessed at a moderately suprathreshold stimulation intensity and marked directly on the scalp with a soft-tip pen. On this site, the resting motor threshold (RMT) was determined as the stimulator intensity needed to produce a response of at least 50 $\mu \mathrm{V}$ in amplitude in the relaxed APB in at least five of ten consecutive stimulations at a resolution of $1 \%$ of the maximal stimulator output (Rossini et al., 1994). Complete muscle relaxation was monitored throughout the experiment and regulated by audiovisual feedback.

\section{Paired associative stimulation}

The PAS protocol exactly reproduces the experimental procedure by Wolters and co-workers (2005), which represented a modification of PAS protocol generally used for the M1 area (Stefan et al., 2002). The protocol consisted of single electrical stimuli delivered to the right $\mathrm{MN}$ at the level of the wrist at $300 \%$ of the perceptual threshold, followed by TMS delivered over the hand representation of S1. Namely, the left S1 was stimulated by placing the central area of the junction of the two coil wings at a scalp site on Cp3. TMS was applied at an intensity of 1.3 times the individual RMT (average $\mathrm{RMT} \pm \mathrm{SE}=38.7 \pm 1.4 \%$ ). The interstimulus interval (ISI) between the MN stimulation and the subsequent TMS pulse was fixed depending on individual N20 latency (Mariorenzi et al., 1991; Ziemann et al., 2004): during the SEPs recording phase of the pre-selection session, the N20 latency of each subject was noticed, and this measure was used as the individual ISI during the PAS protocol (average $\mathrm{ISI} \pm \mathrm{SE}=20.81 \pm 0.16 \mathrm{~ms}$ ). One hundred and forty pairs of stimuli were delivered at $0.1 \mathrm{~Hz}$ over 25 min. During PAS, subjects were asked to keep their right arm completely relaxed and to watch their own right hand, since this condition has been demonstrated to give the maximal PAS-induced plasticity (Stefan et al., 2004). Figure 2 depicts pre- and postPAS SEPs following MN stimulation during the first experimental session (11.00 a.m. of the first day) in a representative subject.

\section{Statistical analysis}

The main dependent variable for the evaluation of the PAS influence on the SEPs after SD was the amplitude of the N20-P25 complex, computed as the difference between N20 and P25 peaks at the Cp3 electrode; we also considered the adjacent $\mathrm{C} 3$ and P3 electrodes. All the collected data were epoched off-line between -32 and $+88 \mathrm{~ms}$ relative to the MN stimulation. Epochs containing artefacts were 


\section{Pre-PAS}

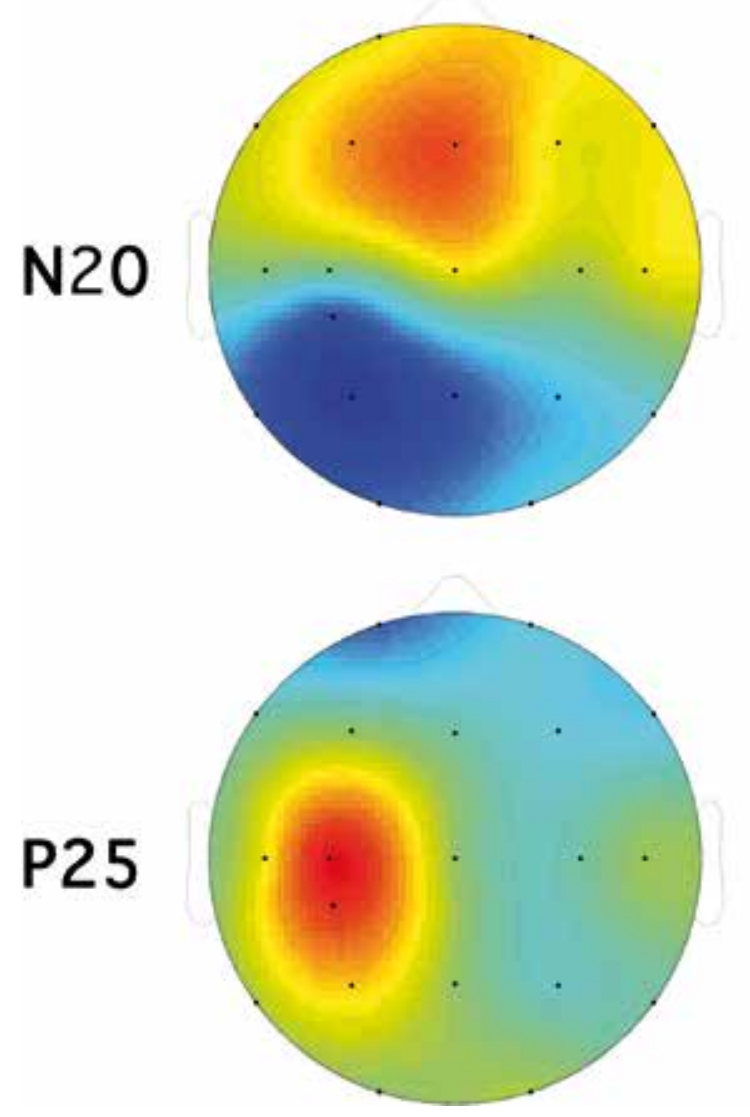

\section{Post-PAS}

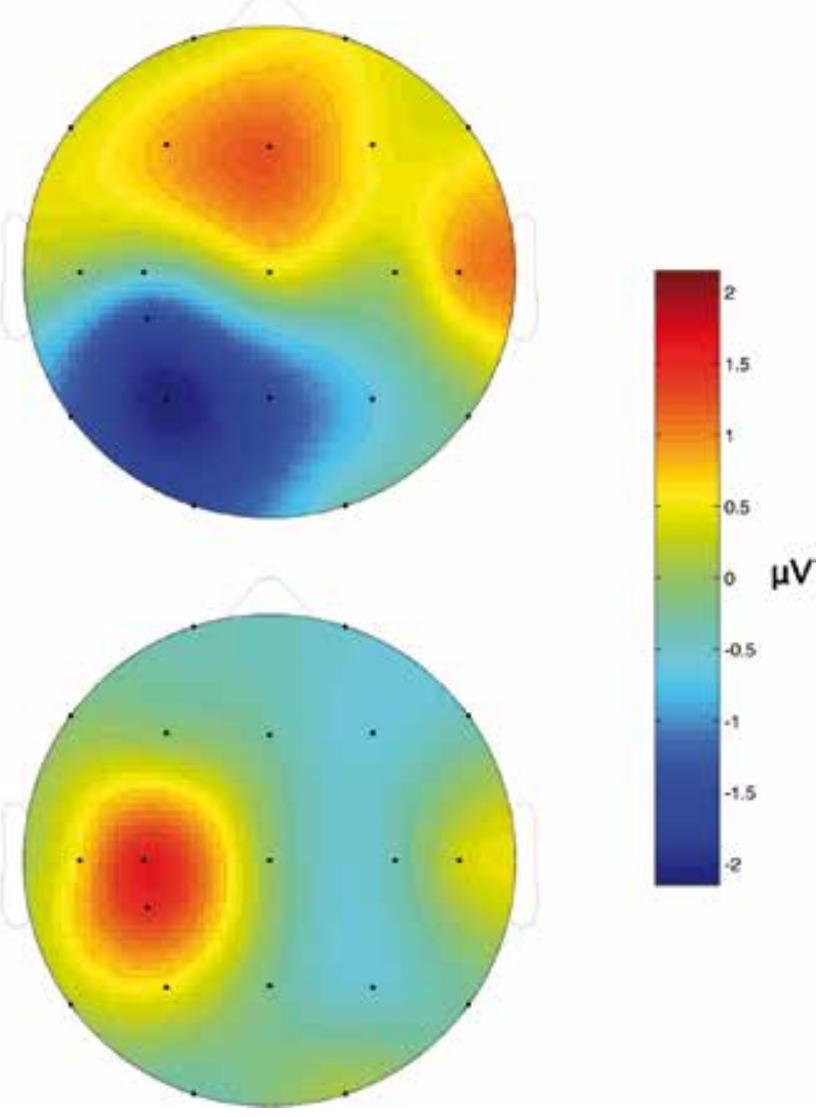

Fig. 3. - Topographic distribution of EEG voltage associated to Pre- and Post-PAS SEPs in correspondence of the $\mathrm{N} 20$ and P25 peaks on Cp3, recorded during the first experimental session. The maps are based on 20 derivations (electrode positions indicated by dots). Values are color-coded and plotted at the corresponding position on the planar projection of the hemispheric scalp model. Values between electrodes were interpolated (biharmonic spline interpolation).

rejected. For each recording, baseline was computed by averaging all the samples between -30 and $-4 \mathrm{~ms}$. Measurement windows were determined by visually inspecting the single SEPs recordings. The N20 amplitude was computed as the difference between the baseline and the first negative peak occurring between 18 and $26 \mathrm{~ms}$ for Cp3, 18 and $24 \mathrm{~ms}$ for C3 and P3 after the MN stimulation. The P25 amplitude was computed as the difference between the baseline and the first positive peak occurring between 22 and $30 \mathrm{~ms}$ for $\mathrm{Cp} 3,20$ and $30 \mathrm{~ms}$ for $\mathrm{C} 3$ and $\mathrm{P} 3$ after the $\mathrm{MN}$ stimulation.

For each experimental session, changes in the SEPs amplitude as a consequence of the PAS protocol were expressed in terms of the ratio between preand post-PAS N20-P25 amplitude. These changes were compared by a two-way repeated measures
ANOVA design, Day (Pre-SD vs. Post-SD) X Time of Day (11.00 a.m. vs. 11.00 p.m.) for the three electrodes. The same ANOVA was performed on the single N20 and P25 components amplitude.

In a previous work (Gorgoni et al., 2014a) we have reported a post-SD amplitude increase of several components (including the P25 but not the N20) of the pre-PAS SEPs recordings shown in the present paper. As a measure of control of the present data, we have now performed the same analysis, that is a two-way repeated measures ANOVA design, Day (Pre-SD vs. Post-SD) X Time of Day (11.00 a.m. vs. 11.00 p.m.) on Cp3, C3 and P3 electrodes, but limited to the post-PAS N20 and P25 components. We expected a SD-induced modulatory effect on post-PAS SEPs similar to that observed in the prePAS SEPs. 

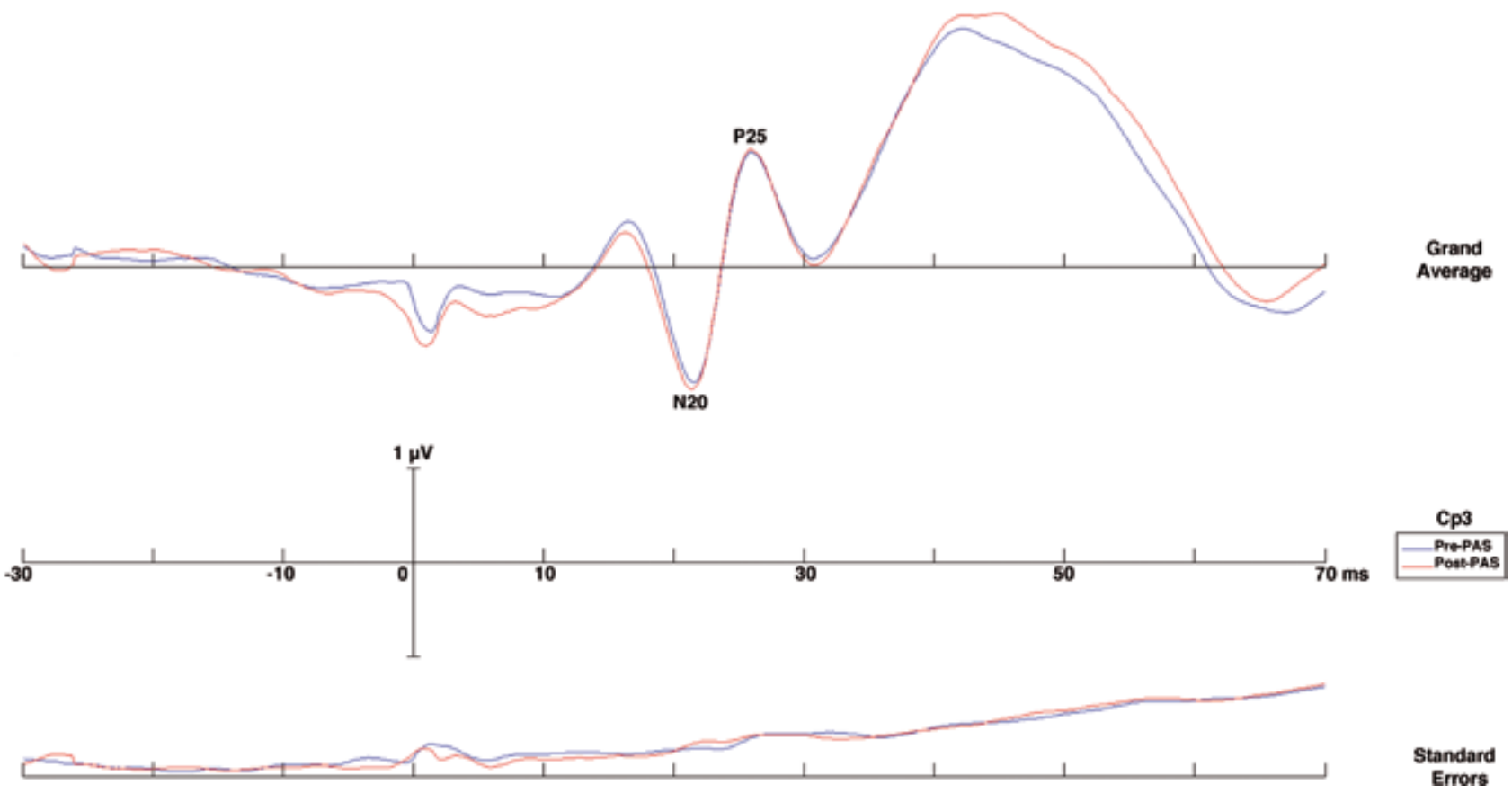

Fig. 4. - Grand average waveforms (top) and corresponding point-by-point standard errors (bottom) of SEPs recorded at $\mathrm{Cp} 3$ following right median nerve stimulation before and after PAS intervention of 16 male subjects during the first experimental session.

\section{Results}

\section{Efficacy of the LTP-like protocol}

Figure 3 depicts the average topographic distribution of EEG voltages associated to the Cp3 N20 and P25 peaks recorded during the first experimental session. Both Pre- and Post-PAS maps show that the N20 is characterized by a negative parietal maximum and a positive frontal maximum contralateral to the peripheral stimulation, while the P25 shows a central positivity contralateral to the peripheral stimulation.

The grand average and the corresponding point-bypoint standard errors of SEPs waveforms recorded on $\mathrm{Cp} 3$ during the first experimental session are illustrated in Figure 4. Although participants were recruited only if they had showed clear LTP-like effects of the PAS protocol during a preliminary evaluation, the PAS intervention failed to induce significant changes in SEPs recorded at Cp3 scalp location within the 20-25 ms time-window in the first experimental session at the 11.00 a.m. of the first day (i.e., without any SD). Indeed, PAS was associated to only a small increase of the voltage: the N20-P25 complex showed an average increase of $2.1 \%$. The amplitude of the N20-P25 complex (mean values are reported in Table 1) was not significantly affected by the associative stimulation $\left(\mathrm{F}_{1,15}=0.78 ; \mathrm{p}=0.39\right)$. This lack of significant changes also extended to the adjacent $\mathrm{C} 3\left(\mathrm{~F}_{1,15}=0.62\right.$; $\mathrm{p}=0.44)$ and $\mathrm{P} 3$ electrodes $\left(\mathrm{F}_{1,15}=0.18 ; \mathrm{p}=0.68\right)$. In fact, only 9 out 16 subjects showed the expected post-PAS N20-P25 amplitude increase at Cp3 during the first experimental session, and in most cases the size or the direction of the PAS effect was not stable between the pre-selection session and the first experimental session (Figure 5).

\section{Changes of pre- vs. post-PAS as a function} of sleep deprivation

Independently of the lack of significant PAS effects in the pre-SD session, we assessed if this measure was affected by SD. Table 2 reports the main effects and the interaction of the ANOVAs performed in order to examine the effect of PAS protocol as a function of SD. The amplitude of the N20-P25 complex recorded at $\mathrm{Cp} 3$ did not show any significant main effect or interaction. Moreover, no significant effect was observed for the single N20 and P25 components. The same lack of significant effects was confirmed at the $\mathrm{C} 3$ and $\mathrm{P} 3$ scalp locations. 


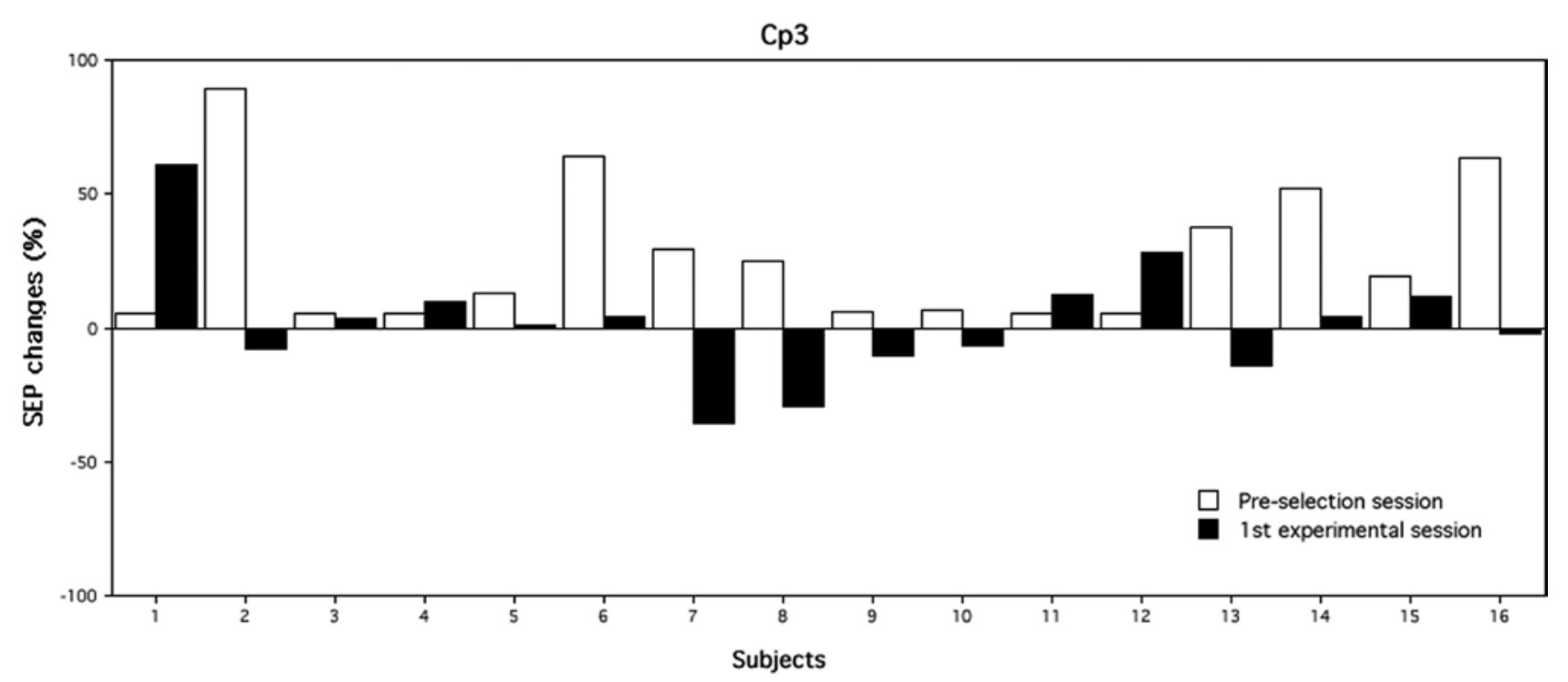

Fig. 5. - Effects of PAS on N20-P25 amplitude of SEPs recorded on Cp3, expressed as a percentage (post-PAS/prePAS) for individual subjects in the pre-selection session (white bar) and first experimental session (black bar). Zero corresponds to the pre-PAS N20-P25 amplitude.

Changes in post-PAS cortical excitability as a function of sleep deprivation

Table 3 shows the main effects and the interaction of the ANOVAs performed with the aim to control the modulatory effects of SD on post-PAS recordings. Results show a significant (FDR $q \leq 0.02 ; P \leq$ 0.05 , corresponds to an $F \geq 4.69$ ) main effect of Day at Cp3, C3 and P3 for the P25, with an amplitude increase after SD (Figure 6). No significant Time of Day main effect or interaction were observed.

\section{Discussion}

To the best of our knowledge, this is the first study to directly examine the effect of SD on somatosensory cortex plasticity in humans. We have used the PAS protocol with the aim to induce LTP-like mechanisms in S1 during SD. No significant SEPs amplitude modifications were found after PAS intervention during the first experimental session (11.00 a.m. of the first day, without SD). This lack of effects could be explained by the large interindividual variability observed in the present study, a problem already found in other PAS experiments (Huber et al., 2008; Pellicciari et al., 2009; Lopez-Alonso et al., 2014). Moreover, a noteworthy intraindividual variability was observed, since subjects showed a mean postPAS N20-P25 amplitude increase of $27.4 \%$ in the pre-selection session, while during the first experimental session only a mean amplitude increase of $2.1 \%$ was found. In fact, many subjects show a lack of stability of the size or the direction of PAS effect between pre-selection session and first experimental session. The lack of intraindividual reliability of the PAS protocol has been also found by Fratello and coworkers (2006). Most studies showing a large intraand interindividual variability of PAS-related effects were aimed to characterize changes in motor cortex plasticity. Current results replicate this observation in the somatosensory cortex.

Another explanation for this lack of stable plasticity effects might be that it is due to the different circadian phase in which the protocol was administered, but against this interpretation militates the lack of any time-of-day effects when comparing morning vs. afternoon sessions.

Despite the absence of the expected LTP-like process during the first experimental session, we have tried to evaluate the possible effect of SD on somatosensory plastic processes. PAS intervention failed to induce significant changes in early SEPs components also in the subsequent experimental sessions (11.00 p.m. of the first day; 11.00 a.m. and 11.00 p.m. of the second day). This lack of significant results should be interpreted in terms of low intraand interindividual reliability shown by the PAS protocol when carried out on the S1 responsiveness, 
Table II. - Main effects and interactions of the Day (D) X Time of Day (T) ANOVAs on post- vs pre- PAS amplitude variations (ratio) of N2O-P25 complex, N20 and P25 components in the scalp locations Cp3, C3, P3.

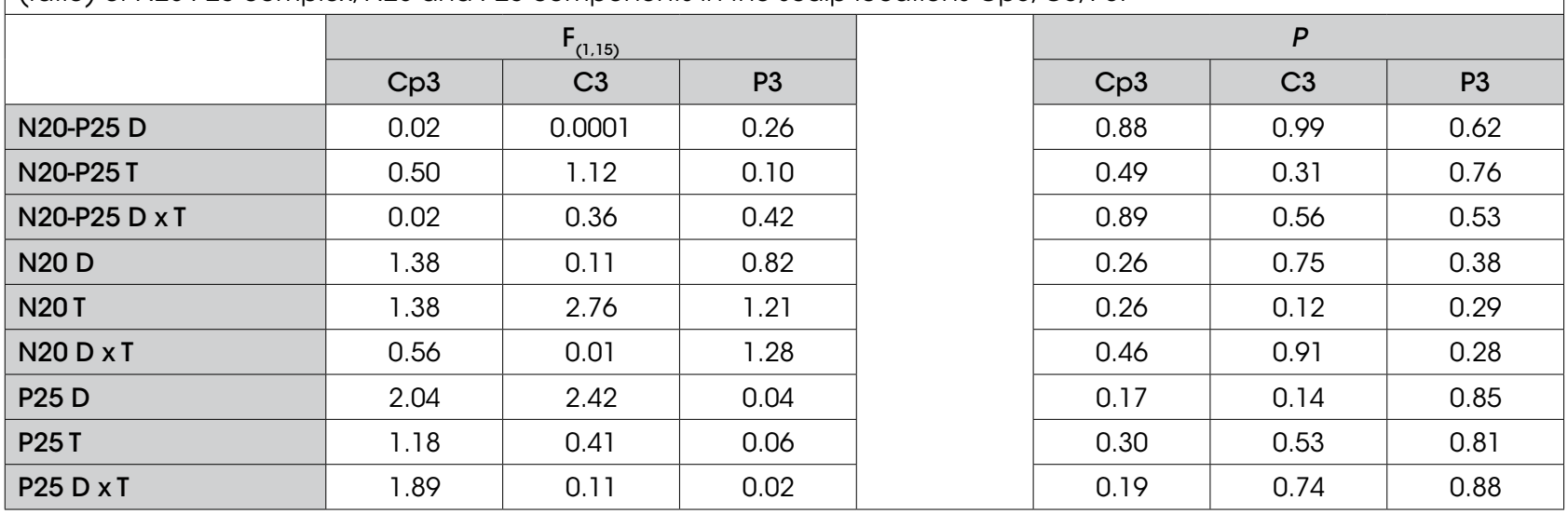

while PAS effects seem to be much more interindividually stable when the motor output is monitored (Tecchio et al., 2008). This result suggests a particular caution when longitudinally evaluating the effect of PAS protocol on cortical plasticity.

SD has induced an amplitude increase in both pre(Gorgoni et al., 2014a) and post-PAS P25 without a circadian influence, while pre- and post-PAS N20 have not been affected by SD. We can conclude, then, that SD has the same modulatory effect on preand post-PAS recordings, excluding the presence of possible bias that can account for the absence of significant results.

A factor potentially affecting the present results depends on our decision to evaluate RMT and perceptual threshold at the beginning of the experiment and fix them to these starting values, instead of adjusting them before each recording. Our decision was driven by the absence of specific information about the relation between LTP-like effects and changes in RMT and perceptual threshold. To the best of our knowledge, when the correlation between PAS-induced plastic effects and RMT has been studied, it was found not significant (Pellicciari et al., 2009; Conde et al., 2012). However, a lack of significant correlations does not exclude a possible contribution of different thresholds across different trials, and the possibility that perceptual and motor thresholds are not similarly affected by SD. For these reasons, we chose to reduce sources of variability, fixing these measures across consecutive recordings.

Furthermore, albeit PAS protocol can be considered an efficient method to induce changes in cortical plasticity (Wischnewski and Schutter, 2015), it is well- known that its effects are influenced by several factors (Ridding and Ziemann, 2010) that may account for the low intra- and interindividual reliability observed in the present study. A possible influence on the present results could be represented by the fluctuation of subjects' attention during the application of the PAS protocol. In fact, PAS effect is enhanced when the experimenter explicitly asks the subjects to pay attention to the stimulated hand (Stefan et al., 2004; Rosenkrantz and Rothwell, 2006), suggesting a noteworthy influence of attention on the efficacy of this method. Albeit in the present experiment we asked the subjects to watch the stimulated hand and to focus attention on it, we have not collected direct measures of attention during the PAS protocol. Intra- and interindividual differences in attention level, then, could have had a role in the observed variability.

Finally, the PAS protocol in our experiment may have been affected by other non-controlled factors, shared with several non-invasive brain stimulation (NIBS) protocols, like cortical thickness (Conde et al., 2012), regular physical activity (Cirillo et al., 2009), genetic polymorphism (Ridding and Ziemann, 2010), or emotional state (Wischnewski and Schutter, 2015). Future efforts should be directed to the systematic research of strategies that can reduce the intra- and interindividual variability in the response to PAS. As well as for others NIBS techniques, the construction of protocols than can account for individual neurophysiological state markers could be considered an objective for the future studies (Karabanov et al., 2015).

Because of the absence of the expected significant effects during the first experimental session, it is dif- 
Table III. - Main effects and interactions of the Day (D) x Time of Day (T) ANOVAs on Post-PAS somatosensory evoked potentials (SEPS) components amplitude (N20, P25) in the scalp locations Cp3, C3 and P3. Significant effects are indicated in bold.

\begin{tabular}{|c|c|c|c|c|c|c|}
\hline & \multicolumn{3}{|c|}{$F_{(1,15)}$} & \multicolumn{3}{|c|}{$P$} \\
\hline & Cp3 & $\mathrm{C} 3$ & P3 & Cp3 & C3 & P3 \\
\hline N20 D & 2.61 & 3.2 & 1.58 & 0.13 & 0.09 & 0.23 \\
\hline N2OT & 0.72 & 0.80 & 1.49 & 0.41 & 0.38 & 0.24 \\
\hline $\mathrm{N} 20 \mathrm{D} \times \mathrm{T}$ & 1.25 & 0.92 & 3.00 & 0.28 & 0.35 & 0.10 \\
\hline P25 D & 5.74 & 4.69 & 6.27 & 0.03 & 0.05 & 0.02 \\
\hline P25 T & 0.55 & 0.36 & 0.99 & 0.47 & 0.56 & 0.33 \\
\hline $\mathrm{P} 25 \mathrm{D} \times \mathrm{T}$ & 0.01 & 0.04 & 1.51 & 0.91 & 0.95 & 0.24 \\
\hline
\end{tabular}

ficult to interpret the results about the influence of SD on cortical plasticity. Huber and co-workers (2013) suggested that increased cortical excitability after prolonged wakefulness could be explained in terms of overall build-up of synaptic strength. We have recently reported evidences of increased excitability in human somatosensory cortex after SD (Gorgoni et al., 2014a), but the existence of alterations of plastic mechanisms during sustained wakefulness, and their possible interaction with post-SD changes of cortical excitability remain open questions.

\section{Conflict of interest}

The authors declare that the research was conducted in the absence of any commercial or financial relationships that could be construed as a potential conflict of interest.

\section{Acknowledgments}

This work was supported by a grant from Ministero della Salute - "Ricerca Finalizzata 2009" (RF-20091528677) to LDG, by a grant from Compagnia di San Paolo - "Programma Neuroscienze 2008/09" (3889 SD/sd, 20088.1300) to LDG and by a grant from Sapienza University of Rome - "Progetti di Ricerca di Ateneo 2010" (C26A10AP8N) to LDG.

\section{References}

Bergmann T.O., Molle M., Marshall L., Kaya-Yildiz L., Born J., Siebner H.R. A local signature of LTPand LTD-like plasticity in human NREM sleep. Eur. J. Neurosci., 27: 2241-2249, 2008.
Bushey D., Tononi G., Cirelli C. Sleep and synaptic homeostasis: structural evidence in drosophila. Science, 332: 1576-1581, 2011.

Campbell I.G., Guinan M.J., Horowitz J.M. Sleep deprivation impairs long-term potentiation in rat hippocampal slices. J. Neurophysiol., 88: 10731076, 2002.

Cirillo J., Lavender A.P., Ridding M.C., Semmler J.G. Motor cortex plasticity induced by paired associative stimulation is enhanced in physically active individuals. J. Physiol., 587: 5831-5842, 2009.

Civardi C., Boccagni C., Vicentini R., Bolamperti L., Tarletti R., Varrasi C., Monaco F., Cantello R. Cortical excitability and sleep deprivation: a transcranial magnetic stimulation study. J. Neurol., Neuros. Psychiatry, 71: 809-812, 2001.

Conde V., Vollmann H., Sehm B., Taubert M., Villringer A., Ragert P. Cortical thickness in primary sensorimotor cortex influences the effectiveness of paired associative stimulation. Neuroimage, 60: 864-870, 2012.

Curcio G., Tempesta D., Scarlata S., Marzano C., Moroni F., Rossini P.M., Ferrara M., De Gennaro L. Validity of the Italian Version of the Pittsburgh Sleep Quality Index (PSQI). Neurol. Sci., 34: 511519, 2013.

De Gennaro L., Marzano C., Veniero D., Moroni F., Fratello F., Curcio G., Ferrara M., Ferlazzo F., Novelli L., Pellicciari M.C., Bertini M., Rossini P.M.. Neurophysiological correlates of sleepiness: a combined TMS and EEG study. Neuroimage, 33: 1277-1287, 2007.

De Gennaro L., Fratello F., Marzano C., Moroni F., Curcio G., Tempesta D., Pellicciari M.C., Pirulli C., Ferrara M., Rossini P.M. Cortical plasticity induced by transcranial magnetic stimulation during wakefulness affects electroencephalogram activity during sleep. PLoS ONE, 3: e2483, 2008. 

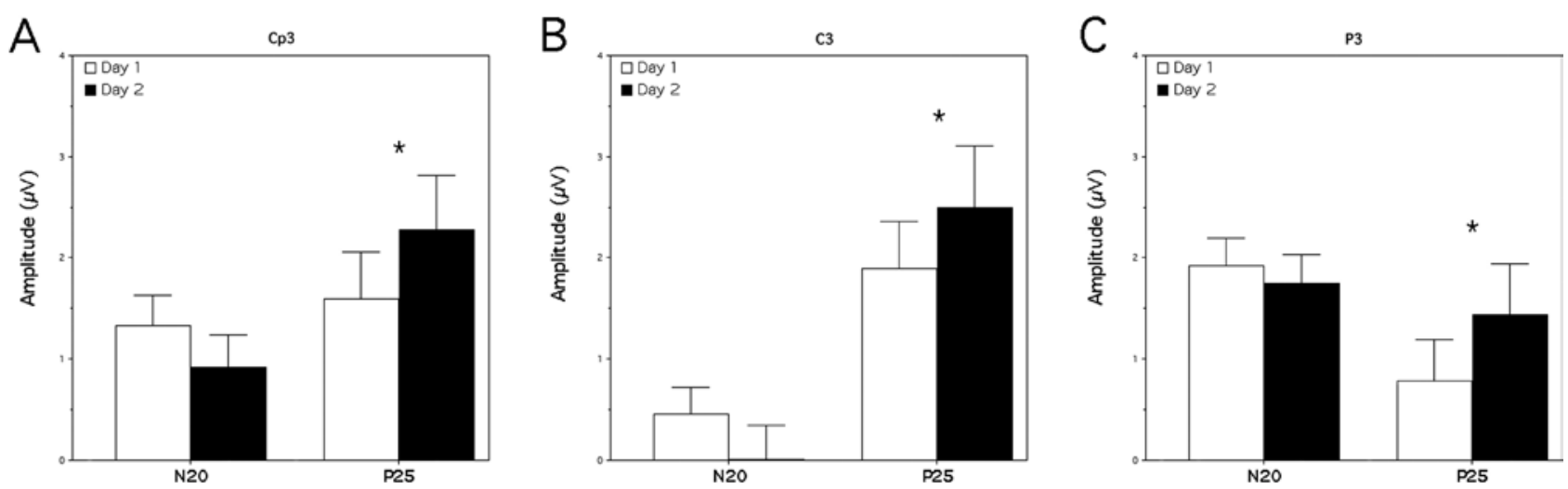

Fig. 6. - Mean amplitude (expressed in $\mu \mathrm{V}$ ) of the Post-PAS somatosensory evoked potentials (SEPs) components $\mathrm{N} 20$ and P25 during the day before (white bars) and after (black bars) sleep deprivation $\left({ }^{*} P \leq 0.05\right.$ ), in the scalp derivation Cp3 (A), C3 (B) and P3 (C). Error bars represent the standard errors.

Di Lazzaro V., Dileone M., Pilato F., Profice P., Oliviero A., Mazzone P., Insola A., Capone F., Ranieri F., Tonali P.A. Associative motor cortex plasticity: direct evidence in humans. Cereb. Cortex, 19: 2326-2330, 2009.

Di Lazzaro V., Dileone M., Profice P., Pilato F., Oliviero A., Mazzone P., Di Iorio R., Capone F., Ranieri F., Florio L., Tonali P.A. LTD-like plasticity induced by paired associative stimulation: direct evidence in humans. Exp. Brain Res., 194: 661-664, 2009.

Donlea J.M., Thimgan M.S., Suzuki Y., Gottschalk L., Shaw P.J. Inducing sleep by remote control facilitates memory consolidation in Drosophila. Science, 332: 1571-1576, 2011.

Durmer J.S. and Dinges D.F. Neurocognitive consequences of sleep deprivation. Semin. Neurol., 25: 117-129, 2005.

Fratello F., Veniero D., Curcio G., Ferrara M., Marzano C., Moroni F., Pellicciari M.C., Bertini M., Rossini P.M., De Gennaro L. Modulation of corticospinal excitability by paired associative stimulation: reproducibility of effects and intraindividual reliability. Clin. Neurophysiol., 117: 2667-2674, 2006.

Gais S., Plihal W., Wagner U., Born J. Early sleep triggers memory for early visual discrimination skills. Nat. Neurosci., 3: 1335-1339, 2000.

Gais S. and Born J. Declarative memory consolidation: mechanisms acting during human sleep. Learn. Mem., 11: 679-685, 2004.

Gorgoni M., Ferlazzo F., Moroni F., D'Atri A., Donarelli S., Fanelli S., Gizzi Torriglia I., Lauri G., Ferrara M., Marzano C., Rossini P.M., Bramanti P., De Gennaro L. Sleep deprivation affects somatosensory cortex excitability as tested through median nerve stimulation. Brain Stim., 7: 732-739, 2014.

Gorgoni M., Ferlazzo F., Ferrara M., Moroni F., D’Atri A., Fanelli S., Gizzi Torriglia I., Lauri G., Marzano C., Rossini P.M., De Gennaro L. Topographic electroencephalogram changes associated with psychomotor vigilance task performance after sleep deprivation. Sleep Med., 15: 1132-1139, 2014.

Huber R., Ghilardi M.F., Massimini M., Tononi G. Local sleep and learning. Nature, 430: 78-81, 2004.

Huber R., Ghilardi M.F., Massimini M., Ferrarelli F., Riedner B.A., Peterson M.J., Tononi G. Arm immobilization causes cortical plastic changes and locally decreases sleep slow wave activity. Nat. Neurosci., 9: 1169-1176, 2006.

Huber R., Esser S.K., Ferrarelli F., Massimini M., Peterson M.J., Tononi G. TMS-induced cortical potentiation during wakefulness locally increases slow wave activity during sleep. PLoS ONE, 2: e276, 2007.

Huber R., Maatta S., Esser S.K., Sarasso S., Ferrarelli F., Watson A., Ferreri F., Peterson M.J., Tononi G.. Measures of cortical plasticity after transcranical paired associative stimulation predict changes in electroencephalogram slow-wave activity during subsequent sleep. J. Neurosci., 28: 7911-7918, 2008.

Huber R., Mäki H., Rosanova M., Casarotto S., Canali P., Casali A.G., Tononi G., Massimini M. Human cortical excitability increases with time awake. Cereb. Cortex, 23: 332-338, 2013.

Karabanov A., Ziemann U., Hamada M., George M.S., Quartarone A., Classen J., Massimini M., 
Rothwell J., Siebner H.R. Consensus paper: probing homeostatic plasticity of human cortex with non-invasive transcranial brain stimulation. Brain Stim., 8: 442-454, 2015.

Kopp C., Longordo F., Nicholson J.R., Luthi A. Insufficient sleep reversibly alters bidirectional synaptic plasticity and NMDA receptor function. J. Neurosci., 26: 12456-12465, 2006.

Korchounov A. and Ziemann U. Neuromodulatory neurotrasmitters influence LTP-like plasticity in human cortex: a pharmaco-TMS study. Neuropsychopharmacology, 36: 1894-1902, 2011.

Kreuzer P., Langguth B., Popp R., Raster R., Busch V., Frank E., Hajak G., Landgrebe M. Reduced intra-cortical inhibition after sleep deprivation: a transcranial magnetic stimulation study. Neurosci. Lett., 493: 63-66, 2011.

Kriváneková L., Lu M.K., Bliem B., Ziemann U. Modulation of excitability in human primary somatosensory and motor cortex by paired associative stimulation targeting the primary somatosensory cortex. Eur. J. Neurosci., 34: 1292-1300, 2011.

Litvak V., Zeller D., Oostenveld R., Maris E., Cohen A., Schramm A., Gentner R., Zaaroor M., Pratt H., Classen J. LTP-like changes induced by paired associative stimulation of the primary somatosensory cortex in humans: source analysis and associated changes in behavior. Eur. J. Neuorsci., 25: 2862-2874, 2007.

Liu Z.W, Faraguna U., Cirelli C., Tononi G., Gao X.B. Direct evidence for wake-related increases and sleep-related decreases in synaptic strength in rodent cortex. J. Neurosci., 30: 8671-8675, 2010.

López-Alonso V., Cheeran B., Río-Rodríguez D., Fernández-del-Olmo M. Inter-individual variability in response to non-invasive brain stimulation paradigms. Brain Stim., 7: 372-380, 2014.

Manganotti P., Palermo A., Patuzzo S., Zanette G., Fiaschi A. Decrease of cortical excitability in human subjects after sleep deprivation. Neurosci. Lett., 304: 153-156, 2001.

Manganotti P., Bongiovanni L.G., Fuggetta G., Zanette G., Fiaschi A. Effects of sleep deprivation on cortical excitability in patients affected by juvenile myoclonic epilepsy: a combined transcranial magnetic stimulation and EEG study. J. Neurol. Neuros. Psychiatry, 77: 56-60, 2006.

Maquet $\mathrm{P}$. The role of sleep in learning and memory. Science, 294: 1048-1052, 2001.

Mariorenzi R., Zarola F., Caramia M.D., Paradiso C., Rossini P.M. Noninvasive evaluation of cen- tral motor tract excitability changes following peripheral nerve stimulation in healthy humans. Electroenceph. Clin. Neurophysiol., 81: 90-101, 1991.

Mascetti L., Muto V., Matarazzo L., Foret A., Ziegler E., Albouy G., Sterpenich V., Schmidt C., Degueldre C., Leclercq Y., Phillips C., Luxen A., Vandewalle G., Vogels R., Maquet P., Balteau E. The impact of visual perceptual learning on sleep and local slow-wave initiation. J. Neurosci., 33: 3323-3331, 2013.

McCoy J.G., and Strecker R.E. The cognitive cost of sleep lost. Neurobiol. Learn. Mem., 96: 564-582, 2011.

McDermott C.M., LaHoste G.J., Chen C., Musto A., Bazan N.G., Magee J.C. Sleep deprivation causes behavioral, synaptic and membrane excitability alterations in hippocampal neurons. J. Neurosci., 23: 9687-9695, 2003.

Murakami T., Sakuma K., Nomura T., Uemura Y., Hashimoto I., Nakashima K. Changes in somatosensory-evoked potentials and high-frequency oscillations after paired-associative stimulation. Exp. Brain Res., 184: 339-347, 2008.

Nitsche M.A., Roth A., Kuo M-F., Fischer A.K., Liebetanz D., Lang N., Tergau F., Paulus W. Timing-dependent modulation of associative plasticity by general network excitability in the human motor cortex. J. Neurosci., 27: 3807-3812, 2007.

Pellicciari M.C., Miniussi C., Rossini P.M., De Gennaro L. Increased cortical plasticity in the elderly: changes in the somatosensory cortex after paired associative stimulation. Neuroscience, 163: 266-276, 2009.

Ridding M.C. and Ziemann U. Determinants of the induction of cortical plasticity by non-invasive brain stimulation in healthy subjects. J. Physiol., 588: 2291-2304, 2010.

Rosenkranz K. and Rothwell J.C. Differences between the effects of three plasticity inducing protocols on the organization of the human motor cortex. Eur. J. Neurosci., 23: 822-829, 2006.

Rossini P.M., Barker A.T., Berardelli A., Caramia M.D., Caruso G., Cracco R.Q., Dimitrijević M.R., Hallett M., Katayama Y., Lücking C.H., et al. Noninvasive electrical and magnetic stimulation of the brain, spinal cord and roots: basic principles and procedures for routine clinical application. Report of an IFCN committee. Electroencephalogr. Clin. Neurophysiol., 91: 79-92, 1994.

Stefan K., Kunesch E., Cohen L.G., Benecke R., Classen J. Induction of plasticity in the human 
motor cortex by paired associative stimulation. Brain, 123: 572-584, 2000.

Stefan K., Kunesch E., Benecke R., Cohen L.G., Classen J. Mechanisms of enhancement of human motor cortex excitability induced by interventional paired associative stimulation. J. Physiol. 543: 699-708, 2002.

Stefan K., Wycislo M., Classen J. Modulation of associative human motor cortical plasticity by attention. J. Neurophysiol., 92: 66-72, 2004.

Stickgold R. Sleep-dependent memory consolidation. Nature, 437: 1272-1278, 2005.

Tecchio F., Zappasodi F., Pasqualetti P., De Gennaro L., Pellicciari M.C., Ercolani M., Squitti R., Rossini P.M. Age dependence of primary motor cortex plasticity induced by Paired Associative Stimulation. Clin. Neurophysiol., 119: 675-682, 2008.

Terney D., Beniczky S., Varga E.T., Kéri S., Nagy H.G., Vécsei L. The effect of sleep deprivation on median nerve somatosensory evoked potentials. Neurosci. Lett., 383: 82-86, 2005.

Tononi G. and Cirelli C. Sleep and synaptic homeostasis: a hypothesis. Brain Res. Bull., 62: 143-150, 2003.

Tononi G. and Cirelli C. Sleep function and synaptic homeostasis. Sleep Med. Rev., 10: 49-62, 2006.

Tononi G. and Cirelli C. Sleep and the price of plasticity: from synaptic and cellular homeostasis to memory consolidation and integration. Neuron, 81: 12-34, 2014.

Vyazovskiy V.V., Cirelli C., Pfister-Genskow M., Faraguna U., Tononi G. Molecular and electrophysiological evidence for net synaptic potentiation in wake and depression in sleep. Nat. Neurosci., 11: 200-208, 2008.
Vyazovskiy V.V., Olcese U., Lazimy Y.M., Faraguna U., Esser S.K., Williams J.C., Cirelli C., Tononi G. Cortical firing and sleep homeostasis. Neuron, 63: 865-878, 2009.

Vyazovskiy V.V., Olcese U., Cirelli C., Tononi G. Prolonged wakefulness alters neuronal responsiveness to local electrical stimulation of the neocortex in awake rats. J. Sleep Res., 22: 264-271, 2013.

Walker M.P. Cognitive consequences of sleep and sleep loss. Sleep Med., 9: S29-S34, 2008.

Walker M.P. and Stickgold R. Sleep-dependent learning and memory consolidation. Neuron, 44: 121-133, 2004.

Wassermann E.M. Risk and safety of repetitive transcranial magnetic stimulation: report and suggested guidelines from the International Workshop on the Safety of Repetitive Transcranial Magnetic Stimulation, June 5-7, 1996. Electroencephalogr. Clin. Neurophysiol., 108: 1-16, 1998.

Wischnewski M. and Schutter D.J. Efficacy and time course of paired associative stimulation in cortical plasticity: implications for neuropsychiatry. Clin. Neurophysiol., 127: 732-739, 2016.

Wolters A., Sandbrink F., Schlottmann A., Kunesch E., Stefan K., Cohen L.G., Benecke R., Classen J. A temporally asymmetric Hebbian rule governing plasticity in the human motor cortex. $J$. Neurophysiol., 89: 2339-2345, 2003.

Wolters A., Schmidt A., Schramm A., Zeller D., Naumann M., Kunesch E., Benecke R., Reiners K., Classen J. Timing-dependent plasticity in human primary somatosensory cortex. J. Physiol., 565: 1039-1052, 2005.

Ziemann U., Iliać T.V., Pauli C., Meintzschel F., Ruge D. Learning modifies subsequent induction of long-term potentiation-like and long-term depression-like plasticity in human motor cortex. J. Neurosci., 24: 1666-1672, 2004. 